\title{
Research on Surface Modification Methods of Nanoparticles
}

\author{
Lin Guomin \\ School of Mechanical Engineering \\ Xijing University \\ Xi 'an, China \\ e-mail: gmlin@21cn.com
}

\author{
Shang Miao \\ School of Mechanical Engineering \\ Xijing University \\ $\mathrm{Xi}$ 'an, China \\ e-mail:445700839@qq.com
}

\author{
Wang Yongfu \\ School of Mechanical Engineering \\ Xijing University \\ $\mathrm{Xi}$ 'an, China \\ e-mail: wangyongfu2000@126.com \\ Xue Mengxuan \\ School of Mechanical Engineering \\ Xijing University \\ $\mathrm{Xi}$ 'an, China \\ e-mail: 719866076@qq.com
}

\begin{abstract}
Surface modification of nanoparticles is a new technology, it refers to the use of physical, chemical and other methods to deal with surface of the nanoparticles. Research objective of this paper is surface modification methods and applications. All analysis is based on the need of use, change the structure and status of the surface of nanoparticles purposefully such as crystal structure of surface, functional groups, surface energy, surface wettability, electric property, surface adsorption and reaction characteristics and so on. The types and purposes of nanoparticle surface modification are introduced. The mechanism, characteristics and process of surface physical modification methods of nanoparticles such as surfactant and surface deposition are analyzed. The characteristics, mechanism and chemical reaction process of the nanoparticles surface chemical modification methods such as esterification reaction, coupling agent, phosphate eater, surface graft modification, in-situ modification are analyzed. Four examples of application are selected to verify the methods above. The verification results show the methods of nanoparticles surface modified are correct.
\end{abstract}

Key words-Nanoparticles;Surface physical modification; Surface chemical modification; Dispersion ; Graft

\section{INTRODUCTION}

Nanomaterials have been called as a new material of the $21 \mathrm{st}$ century, it can be roughly divided into nanopowder (0 dimension), nanofiber (1 dimension), nanofilm (2 dimensions), nanoblock (3 dimensions) and nano composite materials etc. The special type structure of nanoparticles has some special effects such as volume effect, surface effect, quantum size effect and macroscopic quantum tunneling effect, etc., so that the nanomaterial has some special performances in optics, mechanics, electricity, magnetism, superconductivity etc. Nanomaterials are widely used as catalysts, lubricants, building materials, ceramic materials, gas sensitive materials, insulation materials, textile materials, luminescence materials, biomedical materials and so on. So the preparation and properties of nanomaterials becomes one of the research hotspots in recent 20 years.
Surface modification of nanoparticles refers to the use of physical, chemical and other methods to deal with surface of the nanoparticles, according to the need of use, change the structure and status of the surface of nanoparticles purposefully such as crystal structure of surface, functional groups, surface energy, surface wettability, electric property, surface adsorption and reaction characteristics etc. New functions of nanostructure are given, and make its physical properties improved such as particle size, liquidity and electric property. Surface modification of the nanoparticles can achieve the following purpose [1]: to improve or change the dispersion of the nanoparticles; to improve the surface activity of the nanoparticles or compatibility; to improve light resistance, ultraviolet resistance, heat resistance, weather resistance and other performance of the nanoparticles; new functions in physical, chemical, mechanical and other aspects are produced on the particle surface. Surface modification methods of nanoparticles according to its basic principle can be divided into surface physical modification and surface chemical modification. According to the different surface modification agent, surface modification methods of nanoparticles can be divided into inorganic modification and organic modification [2]. Surface modification of the nanoparticles can be implemented after the particles formed or in the process of particle forming. Surface modification process of nanoparticles as shown in Fig .1.

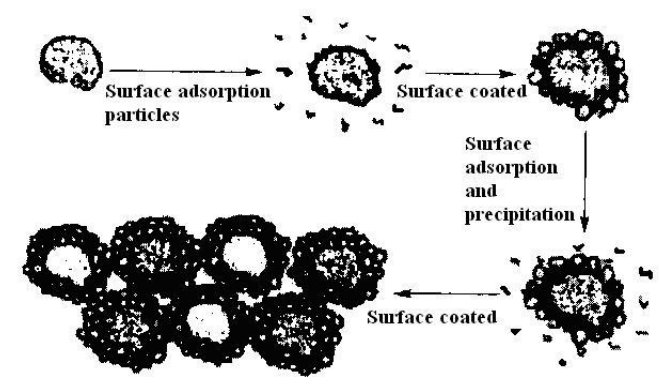

Figure 1. Nanoparticles surface modification process 


\section{SURFACE PHYSICAL MODIFICATION METHODS OF THE NANOPARTICLES}

Surface physical modification of the nanoparticles makes the modifier fixed on the surface of nanoparticles by physical adsorption or surface deposition, it does not change the surface structure of nanoparticles. Surface adsorption has a stability mechanism in electrostatic field and making the dispersion effect strengthening, so as to prevent nanoparticles reunion. Surface sediment can change the surface properties of the particles and reduce the surface activity of nanoparticles to improve the dispersion. Through the physical surface modification, nanoparticles reunion again as strong mixing causes the modifier fall off.

\section{A. Method of surfactant}

Through molecular inter-atomic forces such as Vander Waals force and hydrogen bond would adsorb surfactant to the surface of the nanoparticles as a package of nucleus and formed a coat layer on surface of the core [3], in order to reduce the surface original tension of the nanoparticles and stop reunion between particles for achieving the goal of uniform stable dispersion. There are two kinds of functional groups with completely different features in the surfactant molecules, a kind of polar group is a hydrophilic, another is a non-polar groups with lipophilicity. When inorganic nanoparticles are dispersed in aqueous solution, the surfactant nonpolar pro-oil base will be adsorbed onto the surface of the particles, and polarity hydrophilic group with water soluble, to reach the purpose of inorganic nanoparticles dispersing in water. On the other hand when nanoparticles dispersed in nonpolar oily solution, polar functional groups of the surfactant are adsorbed onto the surface of the nanoparticles, while the nonpolar functional groups mixed with oily medium. Many inorganic oxide or hydroxide nanoparticles have a potential value on the specific surface, the potential value can determine its $\mathrm{PH}$ value in the corresponding solution. Therefore, according to each kind of material surface potential, the $\mathrm{PH}$ value of the solution can be adjusted, and then the surface modification of organic is obtained by adsorption and cladding of the surfactant. For example, dodecyl benzene sulfonic acid sodium as surfactant modification, $\mathrm{Cr}_{2} \mathrm{O}_{3}$ and nanoparticles, make it dispersed stably in ethanol [4].

\section{B. Method of surface deposition}

Using electroless plating method, thermal decomposition-reduction method, coprecipitation method, homogeneous precipitation method, solvent-gel method, hydrolysis synthesis method, the surface cladding layer is formed on the surface of nanoparticles through the deposition reaction. The cladding is fixed on the surface of the particles by other means of processing, so as to improve or change the surface properties of the nanoparticles. New functions of nanoparticles are produced by surface deposition modification, for example, composite nanoparticles of metal/ceramic, which formed by $\mathrm{Cu}$ coating $\mathrm{TiO}_{2}$ prepared through chemical plating surface deposition, have good conductive properties and high strength mechanical properties.

\section{SURFACE CHEMICAL MODIFICATION METHODS OF NANOPARTICLES}

By reaction produced between surface of nanoparticles and modifier, surface structure and state of the nanoparticles are changed to achieve the purpose of surface modification, this is called as surface chemical modification of the nanoparticles. Surface chemical modification methods of nanoparticle include esterification reaction method, coupling agent method, phosphate ester method, surface grafting improvement method and in situ modification method.

\section{A. Esterification reaction method}

The chemical reaction between metal oxide and alcohol is called as esterification reaction. Using the esterification reaction of modification to nanoparticles surface, the original surface with hydrophilic and oleophobic become into the surface with oil-wet hydrophobic. Esterification modification method for nanoparticles surface for weakly acidic or neutral is the most effective. Polycondensation reaction produced between hydroxyl of alcohol and particle surface hydroxyl and took off a water molecule for surface modification. The mechanism of $\mathrm{SiO}_{2}$ modified with alcohol is as follows:

$$
\mathrm{Si}-\mathrm{OH}+\mathrm{R}-\mathrm{OH} \rightarrow \mathrm{Si}-\mathrm{OR}+\mathrm{H}_{2} \mathrm{O}
$$

The $R$ base group of lipophilicity grafted to $\mathrm{SiO}_{2}$ surface, thus wettability increasing of $\mathrm{SiO}_{2}$ nanoparticles and organic matter. In order to promote the reaction is for positive, the key is leads out of the water from reaction system. Experiment shows that using alcohols with titanium dioxide reaction, to make the titanium dioxide has good lipophilicity, more than $C_{4}$ straight chain alcohols must be adopted. When using alcohol with white carbon black, the higher of the esterification degree on the surface of white carbon black, the more hydrophobic. But ester base hydrolysis is easy and thermal stability is poor.

\section{B. Method of coupling agent}

Coupling agent surface modification is use of the groups at the end of molecular react with the surface of the nanoparticles, to form chemical bonds, on the other end of molecular react with polymer or physical wound, the difference inorganic nanoparticles are closely linked with polymer substrate, so as to improve the comprehensive performance of composite materials.

At present the silane coupling agent, titanate coupling agent and aluminum acid ester coupling agent are mainly adopted [5]. Coupling agent is combined with hydroxyl on the surface of the nanoparticles to form mainly the $M-O$ chemical bonds, the nanoparticles surface is covered with a layer of organic molecular film after modification, this film can improve its dispersion properties and rheological properties significantly. The mechanism of nano $\mathrm{SiO}_{2}$ surface modified by silane coupling agent $Y\left(\mathrm{CH}_{2}\right)_{n} \mathrm{Si}(\mathrm{OR})_{3}$ is as follows:

$$
\begin{array}{r}
\mathrm{Y}\left(\mathrm{CH}_{2}\right)_{n} \mathrm{Si}(\mathrm{OR})_{3}+3 \mathrm{H}_{2} \mathrm{O}=\mathrm{Y}\left(\mathrm{CH}_{2}\right)_{n} \mathrm{Si}(\mathrm{OH})_{3}+3 \mathrm{ROH} \\
n \text { is } 1 \sim 3 ; \mathrm{Y} \text { is } \mathrm{NH}_{2}, \mathrm{NHR}, \mathrm{NH}(\mathrm{CH})_{2} \mathrm{NH}_{2}, \mathrm{NCO}, \mathrm{Cl}, \mathrm{S}, \\
\mathrm{RO}, \mathrm{RCOO} ; \mathrm{R} \text { is } \mathrm{CH}_{3}, \mathrm{C}_{2} \mathrm{H}_{5}, \mathrm{CH}_{3} \mathrm{OCH}_{2} \mathrm{CH}_{2} .
\end{array}
$$




\section{Phosphate ester method}

Surface modification of phosphate ester with nanoparticles of calcium carbonate and barium sulfate, it is mainly $\mathrm{Ca}^{2+}$ and $\mathrm{Ba}^{2+}$ on the surface of phosphate and particles, reaction generated of the phosphate deposits or the surface of ion coated. The surface properties of particles are changed and present a hydrophobic. Surface modification mechanism of phosphate ester to calcium carbonate particles is as follows:

$$
\begin{aligned}
& \mathrm{CaCO}_{3}+\mathrm{ROPO}_{3} \mathrm{H}_{2} \stackrel{a}{\longrightarrow} \\
& {\left[\mathrm{ROPO}_{3} \mathrm{H}+\mathrm{CaCO}_{3}\right]^{+}+\mathrm{ROPO}_{3} \mathrm{H}_{2} \stackrel{b}{\longrightarrow}} \\
& \mathrm{Ca}\left(\mathrm{ROPO}_{3} \mathrm{H}_{2}+\mathrm{CO}_{2} \uparrow\right. \\
& \mathrm{CaCO}_{3}+\mathrm{ROPO}_{3} \mathrm{H}_{2} \stackrel{a}{\longrightarrow}\left[\mathrm{ROPO}_{3} \mathrm{H}+\mathrm{CaCO}_{3}\right]^{+} \\
& \stackrel{c}{\longrightarrow} \mathrm{ROPO}_{3} \mathrm{Ca}_{+} \mathrm{CO}_{2} \uparrow \\
& \mathrm{ROPO}_{3} \mathrm{Ca}+\mathrm{H}_{2} \mathrm{O} \stackrel{d}{\longrightarrow} \mathrm{ROPO}_{3} \mathrm{Ca} \cdot \mathrm{H}_{2} \mathrm{O}
\end{aligned}
$$

Phosphate corrosion surface formed calcium phosphate ester cured, closely attached on the base of phosphate ester $R$ outwards is arranged, purpose of modification. When $R$ is small, according to $\mathrm{a}, \mathrm{b}$; When $R$ is bigger, according to $\mathrm{a}, \mathrm{c}, \mathrm{d}$, generate condensate, then it becomes into a solid form slowly. Organic phosphate and phosphate monoester of similar structure are the combination of organic phosphorus in phosphate and alkyl, it is the combination of the phosphate monoester of phosphorus and alkyl. Organic phosphate and hydroxyapatite particle surfaces are combined by chemical bonds.

\section{Surface graft modification method}

Through the chemical reaction, the method of polymer chain is connected to the nanoparticles surface is called the surface graft modification. Due to the polarity of inorganic nanoparticles surface more containing hydroxyl is larger, polarity of particle surface group must be changed in advance before the coated carrying out, and makes the groups grafting on the particle surface the groups can be participating in polymerization or can trigger a role or can stop polymerization, for polymerization by add the polymerization monomer and initiator. Surface graft modification can be divided into three types [6]: A simultaneous of aggregation and surface grafting; the particle surface graft generated by polymerization; the coupling agent grafting method. Surface graft modification method can make full use of inorganic nanoparticles and polymer respective advantages, realize the optimization design, preparation of nanoparticles with new functions. After grafted on the surface of the nanoparticles the dispersion of the nanoparticles in organic solvents and polymer has been greatly increased, for preparation of polymer composite materials with uniform distribution of inorganic nanoparticles. For example, $\mathrm{SiO}_{2}$ nanoparticles, after methyl methacrylate grafte, have long-term stable dispersion in tetrahydrofuran, but all of the nanoparticles will settlement in methanol in short time. This shows that is not at all solution with good long-term dispersion stability after grafting, graft polymer must miscibility with organic solvents for achieving the goal of a stable dispersion. Dispersion of ferrite nanoparticles is good in water after polyacrylamide grafted, and ferrite nanoparticles by polystyrene grafted have good stable dispersion in benzene.

\section{E. In situ modification method}

In situ surface modification of nanoparticles is a new technology of particle synthesis and surface modification in situ synchronization to complete $[7,8]$. Because of the in situ synthesis, soon after nucleation of the surface of the nanoparticles with high surface energy and high active as well as thermodynamic instability, so the polar groups of ultra dispersion stabilizer with dispersion characteristics and stability apply a strong influence on nanoparticles such as adsorption, complexation, chelation. nanoparticles dispersed phase are formed with nanoparticles by dispersion stabilizer anchoring, in order to reduce the high surface energy of nanoparticle, implement the thermodynamic stability and prevent the particles grew up further. The other side of the polarity of dispersion stabilizer can produce molecular chain curl, twining, branched or through each other, thus nano space network of a polar end to inside is formed. The nano space network separates nanoparticles in network center or network space, curing the nanoparticles in a certain position. A small particle size, narrow distribution nanoscale dispersion with characteristics of highly fragmented and long-term stability can be obtained finally. Due to the chemical environment similar, the different nanoscale dispersion prepared by the method above, the nanoparticles can miscibility each other, so the dispersion stability will not be obvious effect. The distinctive features of nanoparticles in situ synthesis method is that nanometer unit formation, particle size controlling, resistance reunion protection, high dispersion of nano unit are once generated in a system for a long time, to improve its performance and simplifies the operation. The production cost caused by nanometer unit preparation, post processing and chemical modification is greatly reduced, so it is an ideal method of surface modification.

\section{EXAMPLES OF NANOPARTICLES SURFACE MODIFICATION}

\section{A. $\mathrm{Al}(\mathrm{OH})_{3}$ coated by $\mathrm{TiO}_{2}$ nanoparticles surface}

Water-soluble aluminium salts reaction liquid such as aluminium sulfate, sodium aluminate, aluminum pure salt are added into solution containing $\mathrm{TiO}_{2}$ nanoparticles. The solution PH value is adjusted, the aluminum salt in reaction liquid with the increase or lower of $\mathrm{PH}$ value, slowly become into $\mathrm{AlOOH}$ or $\mathrm{Al}(\mathrm{OH})_{3}$ colloidal form. In the process of the reaction because of the competition between all other nuclear and heterogeneous nucleation, so the concentration of aluminum compounds is controlled under the condition of lower than all other nuclear. After surface hydroxyl of $\mathrm{TiO}_{2}$ is combined with $\mathrm{Al}(\mathrm{OH})_{3}$ or $\mathrm{AlOOH}$, eventually forming astatic $\mathrm{Al}(\mathrm{OH})_{3}$ coated on the $\mathrm{TiO}_{2}$ particles surface. $\mathrm{TiO}_{2}$ nanoparticles of surface modification coated with alumina can effectively improve the stability and dispersity of the $\mathrm{TiO}_{2}$ nanoparticles, significantly enhance the ability of ultraviolet shielding. 


\section{B. Deposition $\mathrm{Cu}$ on the $\mathrm{TiO}_{2}$ nanoparticles surface}

Palladium chloride is added into the sol containing $\mathrm{TiO}_{2}$ nanoparticles for activation, and then the sol added into the solution of chemical plating copper, after a period of reaction, a layer of metal copper is uniform deposited on $\mathrm{TiO}_{2}$ nanoparticles surface, the plating presents a polycrystalline shell structure. By means of XRD diffraction analysis, $\mathrm{TiO}_{2}$ nanoparticles surface is fully covered by metal elemental copper, the electrical properties is close to pure copper. Composite nanoparticles of $\mathrm{Cu} / \mathrm{TiO}_{2}$ can effectively reduce the density of the original copper material, and the strength, hardness, wear resistance, high temperature mechanical properties of the copper are improved.

\section{Coupling agent modification $\mathrm{SiO}_{2}$ nanoparticles}

Using 4 grams $\mathrm{SiO}_{2}$ nanoparticles is dispersed in 120 $\mathrm{ml}$ of toluene, ultrasonic dispersion under mechanical agitation for 20 minutes. A certain amount of silane coupling agent and propyl silanethree oxygen radicals (MPS) are mixed in $10 \mathrm{ml}$ of anhydrous ethanol, used $\mathrm{HCl}$ to adjust $\mathrm{PH}$ value, then it is mixed with $\mathrm{SiO}_{2}$ nanoparticles. By nitrogen protection, heated to toluene reflux reaction for 1 hour, and then cooled it to room temperature, after centrifugal separation and drying, $\mathrm{SiO}_{2}$ nanoparticles by silane coupling agent the modified is obtained.

\section{CuS nanoparticles coated by oleic acid}

Take $0.02 \mathrm{~mol}$ of oleic acid copper and $0.02 \mathrm{~mol}$ of oleic acid fully mixing with in $100 \mathrm{ml}$ of base oil, through the dry $\mathrm{H}_{2} \mathrm{~S}$ gas the mixture is violent mixed for 1 hour at room temperature, when the color of the base oil from blue gradually turned brown, it shows chemical reaction producing following as

$$
\mathrm{Cu}(\mathrm{OA})_{2}+\mathrm{H}_{2} \mathrm{~S}=\mathrm{CuS}+2 \mathrm{H}(\mathrm{OA})
$$

Thus it shows that the chemical reaction process not only generates inorganic nanoparticles $C u S$, but also generates the surface modification agent of oleic acid $H(O A)$ without by-products $C u S$.

\section{CONCLUSIONS}

Nanotechnology is profoundly changing the human society and life, as an important part of new material of nanometer materials is the material base of nano science and technology, it plays a huge role in promoting to the development of nano science and nano technology $[9,10]$. Nanoparticles surface modification can purposefully change status and surface structure of nanoparticles, it can give new functional and improve physical performance for nanostructures $[11,12]$, so as to meet the needs of the modern new material, new technology. Further study the physical properties, chemical properties of nanomaterials and surface modification of nanoparticles, to promote the development of new materials and the upgrading of traditional industries.

\section{REFERENCES}

[1] Li Fei, Hao Zhenhai, Wang Zhenzhong. Research progress of surface modification of nano powder $[\mathrm{J}]$. Journal of chemical industry in guangdong. 2010 (5), pp. 13-14

[2] Xu zhijun, Chu Ruiqing. Nano materials and nano technology [M] Beijing: chemical industry press, 2010

[3] LIN Zhidong. Nano material basis and application[M]. Beijing Beijing University press, 2010

[4] Sun Yuxiu. The preparation methods and application of nanometer materials [M]. Beijing: China textile press, 2012

[5] Li Jinling. Research of nano-silica surface modification [J] Materials development and application of 2011 (2),pp.18 to 21

[6] Wu Xueli. Carbon nanotube surface graft polymer modified [D]. Lanzhou university. 2010

[7] Tang Shanfa. Gemini surfactant application of nanomaterials [M] Beijing: chemical industry press, 2011

[8] Wang Hong, Huang Chuanjun Li Laifeng. Plasma surface organic polymer in the application of nanometer materials modified [J]. Yunnan Metallurgy (1), pp. 64-67

[9] Qu Ping Bai Haolong, Wang Xuan. Cellulose based 3D nano materials surface modification [J]. China science and technology papers. 2013 (02), pp. 116-119

[10] Qu Ping. Nano fiber/polylactic acid composite material and interface compatibility research [D]. Beijing forestry university; 2013

[11] ChenYujin.Surficial Decoration and Characterization of Microstructure for Low-dimension Nanostruced Materials[D].Haerbin Eigeneering University,2000

[12] Cheng Qianyi,Zhou Ding,Han Baohang. Supramolecular Surface Modification of Carbon Nanomaterials and Their Applicaions[J].2011(9),pp.2062-2070 